\title{
The role of autophagy in microbial infection and immunity
}

This article was published in the following Dove Press journal:

ImmunoTargets and Therapy

6 January 2015

Number of times this article has been viewed

\author{
Mayura Desai' \\ Rong Fang ${ }^{2}$ \\ Jiaren Sun' \\ 'Department of Microbiology \\ and Immunology, ${ }^{2}$ Department \\ of Pathology, University of Texas \\ Medical Branch at Galveston, \\ Galveston, TX, USA
}

Correspondence: Mayura Desai Department of Microbiology and Immunology, University of Texas Medical Branch, 30I University Boulevard, Galveston, TX 77555-1070, USA

$\mathrm{Tel}+$ I 40977249 I I

Fax + I 4097476869

Email madesai@utmb.edu

\begin{abstract}
The autophagy pathway represents an evolutionarily conserved cell recycling process that is activated in response to nutrient deprivation and other stress signals. Over the years, it has been linked to an array of cellular functions. Equally, a wide range of cell-intrinsic, as well as extracellular, factors have been implicated in the induction of the autophagy pathway. Microbial infections represent one such factor that can not only activate autophagy through specific mechanisms but also manipulate the response to the invading microbe's advantage. Moreover, in many cases, particularly among viruses, the pathway has been shown to be intricately involved in the replication cycle of the pathogen. Conversely, autophagy also plays a role in combating the infection process, both through direct destruction of the pathogen and as one of the key mediating factors in the host defense mechanisms of innate and adaptive immunity. Further, the pathway also plays a role in controlling the pathogenesis of infectious diseases by regulating inflammation. In this review, we discuss various interactions between pathogens and the cellular autophagic response and summarize the immunological functions of the autophagy pathway.
\end{abstract}

Keywords: autophagy, xenophagy, antiviral, antibacterial

\section{Introduction}

Autophagy is a cellular degradation pathway by which cytoplasmic cellular constituents are directed to the lysosome. As a regulated catabolic process activated in response to starvation and other cellular stresses, autophagy plays a critical role in the maintenance of cell homeostasis by eliminating and/or recycling unwanted cell products and defunct organelles. It involves a series of dynamic membrane-rearrangement reactions that lead to either a nonselective "bulk" or selective engulfment of cargo by double-membraned autophagosomes, which then fuse with the lysosome, where their content is degraded. ${ }^{1,2}$

Genetic analysis in yeast has identified over 35 components that are required for different steps of the autophagy process, called Atg1-Atg36. Most of these genes have known mammalian counterparts, and many of the core aspects of the process are conserved. ${ }^{3}$ The Atg proteins are grouped under four broad categories according to their function. The pathway is initiated by a protein serine/threonine kinase complex (Atg1/ULK1, Atg13, and Atg17) that responds to upstream signals. This activates the formation of the phagophore by a lipid kinase signaling complex consisting of the class III phosphoinositide 3-kinase (PI3K) VPS34, VPS15, AMBRA1, ATG6/ Beclin1 and ATG14, which together mediate vesicle nucleation. Atg9, an integral membrane protein, provides lipids to the isolation membrane by cycling between distinct subcellular compartments. Vesicle expansion and formation of autophagosomes 
is controlled by two ubiquitin-like conjugation processes: the Atg12-Atg5-Atg16L1 complex formation (mediated by Atg7 and Atg10), and the conjugation of Atg8/LC3 to phosphatidylethanolamine (PE) (mediated by Atg7 and Atg3). Finally, the newly formed autophagosomes fuse with lysosomes in a process that requires recruitment of the small GTPase Rab7 and involves interaction between distinct soluble $N$-ethylmaleimide-sensitive fusion attachment protein receptors (SNAREs) present on the surfaces of autophagosomes and lysosomes. ${ }^{4-6}$

Though it can mediate cell death through self-cannibalization, autophagy is primarily a cell survival mechanism. Similar to apoptosis, it is induced by cell intrinsic signals such as DNA damage, nutrient deprivation, and oxidative stress, and is also regulated through common molecular factors like the Bcl-2 family members and various transcription factors. The mammalian target of rapamycin (mTOR) kinase that controls protein synthesis and nutrient import plays a vital role in the control of autophagy. The mTORC1 complex integrates signals from multiple upstream signaling pathways such as the class I PI3K, the Akt/PKB (serine/threonine protein kinase B), and the Ras-related small GTPases (RAG). The downstream targets of mTORC1 through which it regulates autophagy are ULK1 and ULK2. ${ }^{7-9}$ Alternatively, the energy-sensing kinase adensine monophosphate-activated protein kinase (AMPK) and the tumor suppressor p53 act as positive regulators of autophagy by restraining mTOR under conditions of metabolic and genotoxic stress, respectively. ${ }^{10,11}$ Additionally, the mitogen-activated kinases (MAPKs), extracellular signal regulated kinase (ERK), p38, and c-Jun N-terminal kinase (JNK), which mediate responses to extracellular stimuli, also induce autophagy in response to various stresses. The anti-apoptotic factors of the Bcl-2 protein family (Bcl-2, Bcl-xl) act as repressors of the Beclin1-VPS34 complex. In response to specific activator signals, the phosphorylation of Bcl-2 by JNK1 decreases the interaction between Bcl-2 and Beclin 1 and triggers autophagy. Equally, death signals, pathogens, and hypoxia that activate factors such as DAP kinase, TRIF/MyD88, and BNIP3, respectively, also disrupt the Bcl-2-Beclin1 interaction to induce autophagy. Further, autophagy is also induced by the stress-related eukaryotic initiation factor $\alpha$ (eIF $\alpha)$ kinase pathway. ${ }^{7,12}$ To survive endoplasmic reticulum (ER) stress, cells mount an unfolded protein response (UPR) to decrease the ER protein load and enhance protein-folding capacity. The UPR is triggered through the activation of ER stress transducers inositolrequiring kinase 1 (IRE1), activating transcription factor 6
(ATF6), and PKR-like ER kinase (PERK) that dissociate from the folding chaperone BiP to induce downstream signaling pathways. While various studies link autophagy to UPR, the induction signal appears to be cell-type-dependent. ${ }^{13}$

Autophagy has been implicated in various physiological processes, and perturbations in the autophagy response are associated with different disease pathologies, including cancer, neurodegeneration, cardiovascular complications, and microbial infection. During the past decade, evidence for the role of autophagy in functions of innate and specific immunity, such as pathogen clearance, lymphocyte development, antigen presentation, and immunoglobulin production, have been described, thereby providing a link between autophagy and the control of microbial infections. ${ }^{14-17}$ Furthermore, evidence has been presented describing strategies that are used by pathogenic microorganisms to overcome or manipulate the autophagy response to prevent their clearance, allowing them to establish infection. Alternatively, virus pathogens have been shown to utilize autophagic structures for virus particle assembly and possibly egress. ${ }^{15,18}$ In this review, we summarize some of the interactions between pathogens and the autophagy pathway and discuss the pro- and antipathogenic ramifications of the process. A brief overview of the pathogen-autophagy interplay is provided in Table 1 .

\section{Virus interactions with autophagy}

Being obligate intracellular pathogens, viruses depend on the host cellular apparatus for their survival and replication. Conceivably, given the importance of autophagy in diverse cellular functions, many viruses have evolved to exploit the cellular autophagy machinery for their survival and replication. On the other hand, the autophagy pathway has emerged as a key effector as well as regulator of antiviral immunity. The following section offers an overview of the interplay between autophagy and viruses.

\section{Proviral autophagy}

While viruses have evolved to subvert the autophagy pathways, some have also devised mechanisms to manipulate the autophagy machinery for their replication cycles. This is achieved either through deregulation of host stress responses and/or by blocking the autophagic flux ${ }^{2,19,20}$ (Figure 1). One of the best studied viruses in this regard, the hepatitis $\mathrm{C}$ virus, triggers autophagy for its replication cycle. At the very outset, the virus induces autophagy to enhance translation of the incoming RNA. ${ }^{2}$ Further, as the ER responds to an overload of viral proteins generated through translation of viral RNA, it leads to a UPR. Several UPR modulators, 
Table I Summary of pathogen interactions with the cellular autophagy machinery

\begin{tabular}{ll}
\hline Pathogen & Interaction with autophagy \\
\hline Hepatitis C virus (HCV) & $\begin{array}{l}\text { Triggers autophagy through endoplasmic reticulum (ER) stress and blocks autophagic flux to enhance viral RNA } \\
\text { translation and replication in autophagic-membrane-associated compartments. Virus-induced mitophagy protects } \\
\text { infected cells from apoptosis. }{ }^{20-23}\end{array}$ \\
Viral 2BC enhances lipidation of LC3 and 3A inhibits autophagosome movement along microtubules to establish a \\
Polio virus (PV) & replication compartment. Virus exits the host cell by an autophagy-related secretary pathway. \\
& Virus induction of autophagy and regulation of autophagic flux enhances virus replication and maximizes dissemination. \\
Coxsackie B (CBV) & Proteolytic cleavage of viral HA increases autophagy, while M2 inhibits autophagosome maturation, compromising \\
Influenza A virus (IAV) & survival of host cells. M2 also promotes relocalization of LC3 to the plasma membrane to support filamentous \\
& budding of virions. Autophagy in dying IAV-infected cells potentiated IAV Ag presentation by DCs to MHC class \\
& I-restricted cytotoxic T lymphocytes.,32-35 \\
Japanese encephalitis & While autophagy proteins play a proviral role in virus replication, conventional autophagy may be antiviral for the virus.
\end{tabular}

virus (JEV)

Human immunodeficiency

virus (HIV)

Virus upregulates autophagy during primary infection and viral Nef blocks autophagosome acidification through Beclin I interaction. Autophagy is essential for Gag processing. In CD4+ $\mathrm{T}$ cells gP4I fusion activity induces autophagy. Alternatively, during the productive phase Nef interacts with IRGM to inhibit autophagy. TLR7/8 activation in virusinfected cells induces autophagy, $2,20,38,39,69$

Hepatitis B virus (HBV) Viral HBx induces autophagy to promote viral DNA replication and envelopment and blocks autophagic degradation through repression of lysosomes. . $^{38,40-43}$

Epstein-Barr virus (EBV) In the latent phase of infection the virus induces autophagy to counter the ER stress-related apoptotic factors, while during the lytic phase autophagosomes are hijacked to promote virus production. The EBNAI protein is presentated on MHC class II through autophagy. ${ }^{7,44-47}$

Herpes simplex virus (HSV) Viral ICP34.5 attenuates autophagy by binding Beclin I and through inhibition of the PKR-elF2 $\alpha$ pathway. In the late-stage of infection viral Us I I inhibits elF2 $\alpha$ phosphorylation. Autophagy is required for MHC class II cross-presentation of viral Ags by dendritic cells (DCs). Viral capsid Ag processing is impaired by the ICP34.5 inhibition of autophagy. $744,48,49,77$

Human cytomegalovirus (HCMV) Inhibits autophagy through activation of the mammalian target of rapamycin (mTOR) pathway. MHC class I

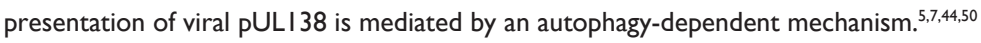

Kaposi's sarcoma-associated herpesvirus (KSHV) Listeria monocytogenes

Virus encoded homologues of Bcl-2 and FLIP interact with Beclin I and Atg3 respectively, leading to inhibition of autophagy to enhance viral proliferation., ${ }^{7}$

Activation of NLRs induces autophagic sequestration of invading bacteria. Additionally, bacterial pore-forming toxin LLO also induces autophagy via mTOR inhibition. On the other hand, LLO and actin polymerization protein ActA prevent entrapment of bacteria in autophagosomes. Also, bacterial phospholipase $C$ enzymes mediate autophagy evasion through disruption of autophagosome inner membrane. The bacterial PRR, PGRP-LE, mediates autophagic targeting of bacteria in Drosophila melanogaster. ${ }^{52-54,61-63,71,72}$

Group A Streptococcus (GAS) Bacteria enter host cells through endocytosis and are susceptible to xenophagic killing. ${ }^{58}$

Mycobacterium tuberculosis Bacteria block phagosome maturation and induction of autophagy facilitates phagosome-lysosome fusion. IFN- $\gamma$ induced autophagy mediates bacterial clearance. ${ }^{59,60,74}$

Rickettsia conorii Bacteria are susceptible to IFN- $\gamma$-induced autophagy. ${ }^{74}$

Salmonella enterica serovar typhimurium

Coxiella burnetti NOD2-mediated autophagy in DCs is required for the generation of CD4+ $4^{+}$-cell responses during bacterial infection. ${ }^{81}$

Anaplasma phagocytophilum

Toxoplasma gondii

Bacteria survive in Coxiella-replicative vacuoles that are decorated with LC3 and Beclin I and inhibition of autophagy impairs bacterial replication. ${ }^{65,66}$

Ats-I hijacks the Beclin I-Atg I4L autophagy initiation pathway. Stimulation of autophagy facilitates infection by providing bacteria access to host cytosolic nutrients. ${ }^{67,68}$

CD40 ligand induces autophagy-mediated fusion of bacteria-containing phagosomes with lysosomes through CD40 signaling. ${ }^{74}$

Chlamydia species

Escherichia coli

Autophagy plays a role in preprocessing of intracellular bacterial Ags before loading onto recycling MHC I complexes. ${ }^{5}$ $\mathrm{CD}^{+} \mathrm{T}$-cell responses are generated through NOD2-mediated autophagy in DCs. Suppression of prolonged NFK-B activation in infected macrophages leads to upregulation of autophagy and promotes cell survival. ${ }^{4,81}$

Abbreviations: NLR, NOD-like receptors; DCs, dendritic cells; IFN- $\gamma$, interferon- $\gamma$; PRR, pattern recognition receptor; PGRP, peptidoglycan-recognition protein; LLO, listeriolysin O; HA, hemagglutinin.

eg, PERK, IRE1 $\alpha$, CCAAT/enhancer-binding proteinhomologous protein (CHOP), and ATF6, seem to play a role in $\mathrm{HCV}$-induced autophagy. ${ }^{21}$ It has been proposed that the HCV-induced autophagic membrane may be used as a membrane-associated compartment for the replication of viral RNA, as the viral NS5A, NS5B, proteins, and nascent viral RNA are colocalized with the autophagosome. ${ }^{21,22}$ Likewise, viral NS4B and the envelope glycoproteins E1 and E2 are also said to contribute to ER stress activation. In addition to its pivotal role in viral RNA replication, 


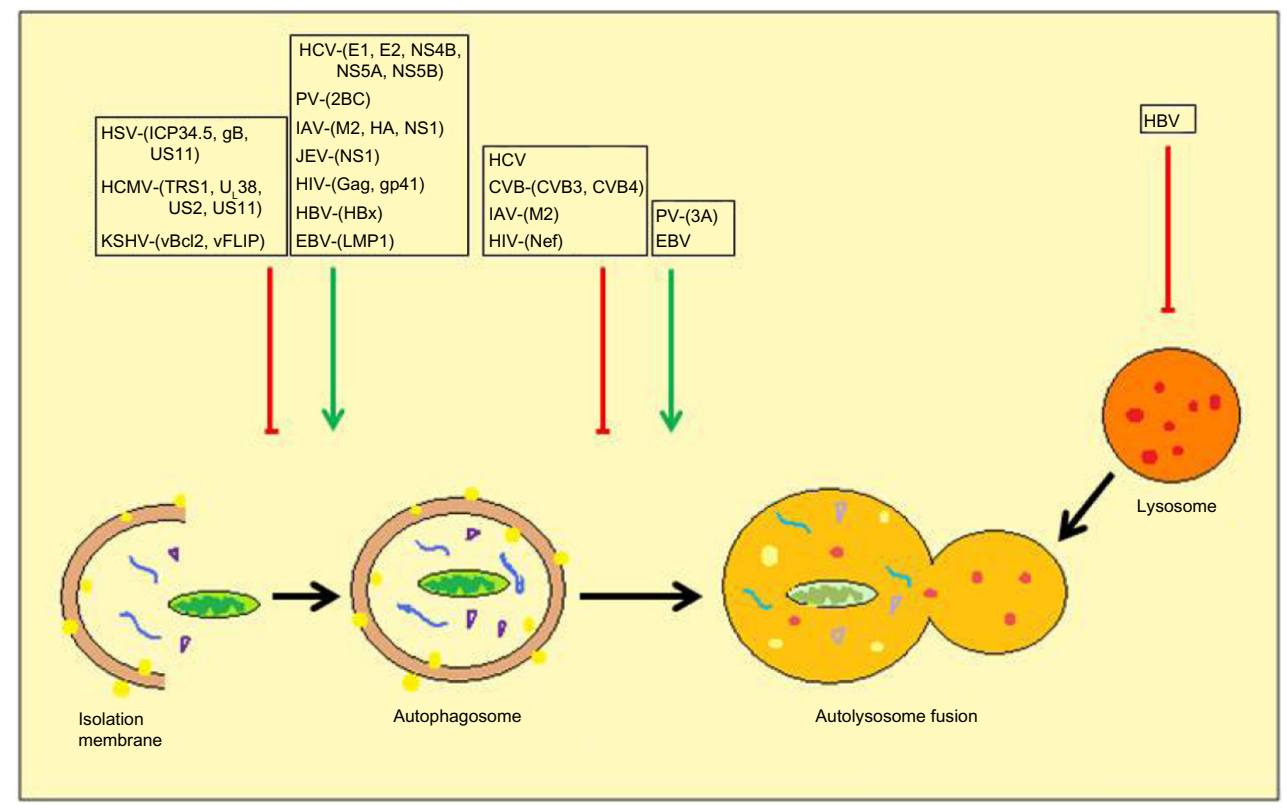

Figure I Virus manipulation of the cellular autophagy pathway.

Notes: Viruses have evolved to either activate (green arrows) or inhibit (red lines) different stages of the autophagic response. Many viral proteins interact with components of the autophagy machinery or modulate the autophagy-related signaling pathways for their survival and/or replication.

Abbreviations: HCV, hepatitis C virus; PV, poliovirus; CVB, Coxsackievirus B; IAV, Influenza A virus; JEV, Japanese encephalitis virus; HIV, human immunodeficiency virus; $\mathrm{HBV}$, hepatitis B virus; EBV, Epstein-Barr virus; HSV, herpes simplex virus; HCMV, human cytomegalovirus; KSHV, Kaposi's sarcoma-associated herpesvirus.

autophagy was shown to regulate the assembly of infectious virions and protection of infected cells from death. ${ }^{22}$ Several autophagy proteins, such as Beclin1, LC3, Atg4B, Atg5, Atg7, and Atg12, are deemed necessary for productive $\mathrm{HCV}$ infection. ${ }^{20}$ Moreover, autophagy proteins also contribute to $\mathrm{HCV}$ particle assembly and/or egress. ${ }^{22} \mathrm{HCV}$ also induced mitochondrial fission and mitophagy to attenuate apoptosis and possibly facilitate viral persistence. ${ }^{23}$

The poliovirus (PV) induces autophagy while inhibiting autophagosome degradation. ${ }^{24}$ Autophagy can be induced by the combination of two viral proteins, termed $2 \mathrm{BC}$ and $3 \mathrm{~A}$. The virus $2 \mathrm{BC}$ increases the lipidation of $\mathrm{LC} 3$, and $3 \mathrm{~A}$ inhibits autophagosome movement along microtubules to block autophagosome-lysosome fusion.,25 The incomplete autophagic process allows the virus to establish a replicative niche within the cytoplasm. ${ }^{26}$ According to one model, the PV replication complex is initially present inside single membrane vesicles, which eventually morph to autophagosomes and amphisomes. The vesicle acidification is critical for the virus life cycle, as the acidic amphisomes promote the late, post-RNA replication step of PV particle maturation. ${ }^{27}$ Further, the virus exits the cell by an autophagy-related secretary pathway. ${ }^{28}$ On the other hand, the related picornavirus, Coxsackievirus B, induces autophagy through its CVB3 and CVB4 proteins. $^{2}$ Further, CVB3 regulates the autophagic flux by inhibiting the maturation of autophagosomes. ${ }^{29}$
Recently, it has been demonstrated that the virus exits the host cell in shed microvesicles displaying autophagosomal markers. ${ }^{30}$ It is known that viruses exploit cellular microvesicle pathways to maximize dissemination. ${ }^{31}$

Influenza A virus (IAV) subverts autophagy by mimicking a host short-linear protein-protein interaction motif. The ability of IAV to evade autophagy depends on the Matrix2 (M2) ion-channel protein. The cytoplasmic tail of IAV M2 interacts directly with the essential autophagy protein LC3 and promotes LC3 re-localization to the unexpected destination of the plasma membrane. LC3 binding is key for virion stability and filamentous budding. ${ }^{32}$ Proteolytic cleavage of the influenza hemagglutinin (HA) protein also increases autophagy. On the other hand, the viral NS1 stimulates autophagy indirectly by upregulating the synthesis of HA and M2. ${ }^{33}$ Moreover, by interacting with Beclin1, M2 blocks autophagic flux through inhibition of autophagosome maturation. ${ }^{34}$ However, inhibition of autophagosome maturation compromises survival of IAV-infected cells, thereby enhancing the proapoptotic effect of the viral protein PB-F1. ${ }^{35}$

The cellular autophagy process is also involved in the early stages of the Japanese encephalitis virus (JEV) infection, and the inoculated viral particles traffic to autophagosomes for subsequent steps of viral infection. Viral replication was seen to be reduced in cells with downregulated Atg5 or Beclin1 expression, which is suggestive of a pro-viral role of 
autophagy in JEV replication. ${ }^{36}$ Conversely, a recent study indicated that, while autophagy is primarily antiviral for JEV, nonlipidated LC3 plays an important autophagy-independent function in the virus life cycle. This was implied as colocalization of the viral nonstructural protein NS1, and LC3 was observed even in Atg5-deficient cells that contain only the nonlipidated form of LC3. ${ }^{37}$

The human immunodeficiency virus (HIV) subverts autophagy to promote its own replication. About ten autophagy genes have been linked to HIV replication. These include four genes involved in the nucleation and elongation of autophagosomes (Atg7, LC3, Atg12, Atg16L2) and two involved in lysosomal function (CLN3 and LAPTM5) that are essential for HIV replication. ${ }^{38}$ Silencing of Beclin1 and Atg5 in macrophages and Beclin1 and Atg7 in monocytes has been shown to inhibit HIV replication. Autophagy is said to be involved in HIV Gag processing, and Gag-derived proteins colocalize with LC3B-II enriched membranes, suggesting a role of autophagy in the production of nascent virions. ${ }^{20,39}$ While the virus upregulates autophagy during the initial stages of primary infection, HIV needs to control the antiviral proteolytic and degradative late stages of autophagy to avoid its self-degradation. The viral Nef protein blocks autophagosome acidification by interacting with Beclin 1 and sequestering Beclin1 to the Golgi complex. ${ }^{2,20,39}$ Further, the immunity-associated GTPase (IRG) family $\mathrm{M}$, which interacts with Atg5 and Atg10, has been reported to be another target of Nef for the accumulation of autophagosomes and HIV-1 production. ${ }^{38}$ In the case of HIV infection of $\mathrm{CD}^{+}$ $\mathrm{T}$ cells, there is an initial induction of autophagy associated with the fusion activity of the HIV gp41 protein. However, during productive infection autophagy is inhibited. ${ }^{39}$

The hepatitis B virus (HBV) induces autophagy to favor its own replication. The exact steps impacted are not clear, but it appears that autophagy either enhances viral DNA replication or facilitates envelopment. The viral HBX protein, a multifunctional protein, has been shown to induce autophagy either through upregulation of PI3KC3 or through upregulation of Beclin1 expression. ${ }^{38} \mathrm{HBX}$ also induced autophagy through the dephosphorylation/activation of the death-associated protein kinase (DAPK).$^{40}$ Additionally, HBX has a repressive effect on lysosomal function, which results in the inhibition of autophagic degradation, and this may be critical to the development of HBV-associated hepatocellular carcinoma (HCC). ${ }^{41}$ Besides this, HBV also activates the ER-associated degradation (ERAD) pathway, which, in turn, reduces the amount of envelope proteins, possibly as a mechanism to control the level of virus particles in infected cells and facilitate the establishment of chronic infections. The ER degradation-enhancing mannosidase-like proteins (EDEMs) are thought to play an important role in relieving ER stress during UPR. Synthesis of EDEMs is significantly upregulated in cells with persistent or transient HBV replication. ${ }^{42}$ Further, mutation analysis showed that the HBV small surface protein (SHBs) could also induce UPR, and the blockage of UPR signaling pathways abrogated the SHB-induced lipidation of LC3-I. This response was required for $\mathrm{HBV}$ envelopment but not for the efficiency of HBV release. ${ }^{43}$

The Epstein-Barr virus (EBV) establishes its latent phase of infection by regulating both the UPR and autophagy. ${ }^{44}$ During its lytic phase, the virally encoded transcription factor Rta induces autophagy via the ERK pathway to promote replication, ${ }^{45}$ and, in the latent phase, the latent membrane protein, LMP1, oncogene of EBV, induces UPR by activating PERK, ATF6, and IRE- $1 .{ }^{46}$ To counter the proapoptotic effects of prolonged PERK activation, LMP-1 activates autophagy. This activation, in turn, promotes degradation of the high levels of LMP1 to reset the cell's physiology to drive proliferation and further increase expression of LMP1. Thus, through a cyclic induction of UPR and autophagy, the cell maintains a supraphysiological level of the LMP1 oncogene. This control of UPR promotes XBP-1-spliced RNA, a plasma cell differentiation factor, and the subsequent secretion of immunoglobulins. ${ }^{45,46}$ Conversely, during the lytic phase of infection, EBV blocks autolysosome fusion to hijack the autophagic vesicles for its intracellular transportation in order to enhance viral production. ${ }^{47}$

\section{Viral attenuation of xenophagy}

Xenophagy is a form of autophagy that specifically targets intracellular pathogens for lysosomal degradation. Viruses regulate xenophagy to evade immune detection and often to promote survival during latency ${ }^{48}$ (Figure 1). A virus family that has developed diverse mechanisms to antagonize the cellular autophagic processes is Herpesviridae. ${ }^{7}$ In the case of the herpes simplex virus (HSV) 1, the virus subverts host autophagy through multiple mechanisms. The neurovirulence factor ICP34.5 blocks the translation repression of PKR by activating a cellular phosphatase PP1 $\alpha$ that mediates the dephosphorylation of eIF $2 \alpha$. The PKR-eIF $2 \alpha$ pathway positively regulates autophagy, and its inhibition ensures efficient translation and accumulation of viral proteins. ${ }^{44}$ In addition, ICP34.5, a multifunctional protein, attenuates autophagy by binding with Beclin1 and also blocks the UPR. The inhibition of autophagy through binding of Beclin1 is said to be 
linked to PKR. ${ }^{7}$ Further, the viral glycoprotein B $(\mathrm{gB})$ has also been shown to block the activation of PERK in infected cells with experimentally induced UPR. PERK inhibition not only controls UPR but also blocks the synthesis of CHOP, thus preventing apoptosis of infected cells. ${ }^{44}$ Another protein Us11, which is synthesized at the late stage of HSV1 infection, like ICP34.5, also inhibits phosphorylation of eIF2 $\alpha$. Thus the two proteins act in concert to regulate the turnover of viral proteins during infection. ${ }^{49}$

The human cytomegalovirus (HCMV) counteracts autophagic degradation in the late stage of infection through its TRS1 protein. Previously, it was shown that TRS1 could neutralize the PKR antiviral effector molecule. ${ }^{7}$ However, later the PKR-binding domain of TRS1 was seen to be dispensable to its inhibitory effect. Subsequently, it was demonstrated that TRS1 interacts with -Beclin1 to inhibit autophagy. ${ }^{50}$ Additionally, HCMV could activate the mTOR signaling pathway in primary human fibroblasts, and rendered infected cells resistant to rapamycin-induced autophagy. Moreover, the infected cells also became resistant to the stimulation of autophagy by lithium chloride, an mTOR-independent inducer of autophagy. It was suggested that the viral early gene $U_{L} 38$ might play a role in the mTOR signaling. ${ }^{7}$ Additionally, two viral gene products, US2 and US11, were reported to bind the chaperone BiP, which is diagnostic of UPR. The virus uses BiP for its assembly. ${ }^{44}$

The $\gamma$-herpesvirus, Kaposi's sarcoma-associated herpesvirus (KSHV), encodes viral homologues of the cellular proteins Bcl-2 and FLIP (FLICE-like inhibitor protein). The KSHV Bcl-2 inhibits autophagy by interacting with Beclin1. Moreover, contrary to the cellular Bcl-2-Beclin1 interaction, which can be modulated by nutrient conditions, the viral $\mathrm{Bcl} 2$ associates with cellular Beclin1 irreversibly, resulting in a steady repression of autophagy in virus-infected cells, which could promote oncogenic events. ${ }^{7}$ The viral FLIP (vFLIP) represses autophagy by interacting with Atg3 to inhibit the conjugation of LC3, which is essential for autophagic membrane biogenesis. This inhibition of autophagy is said to enhance KSHV proliferation. ${ }^{19}$

\section{Autophagy and bacterial infection}

The first observation of infection-triggered autophagy was described in an infection with intracellular bacteria. ${ }^{51}$ Autophagy not only plays a role in bacteria recognition and restriction but also has many other functions in the immune system. Here, we focus on molecular mechanisms of autophagic recognition, targeting and elimination of intracellular bacteria, and manipulation of autophagy by bacteria.

\section{Autophagy induction by bacteria}

Listeria monocytogenes is one of the best studied examples of bacterial induction of autophagy. ${ }^{52}$ Upon entry into host cells, L. monocytogenes rapidly escapes, by using the pore-forming toxin listeriolysin O (LLO) from its phagosome into the cytosol, where it is able to replicate proficiently. ${ }^{53}$ Once in the cytosol, wild-type L. monocytogenes recruit LC3 to bacteria, and at 1 hour post-infection, a population of 37\% intracellular bacteria colocalizes with this autophagy marker. ${ }^{52}$ This level of LC3 recruitment does not occur during infection by an hly (the gene encoding LLO) deletion strain, which points to the possible requirement of LLO for induction of autophagy. Further studies have demonstrated that LLO can activate AMPK and thereby downregulate mTORC1, a control node in the regulation of starvation-induced autophagy ${ }^{54}$ (Figure 2).

In addition, bacterial pathogen-associated molecular patterns (PAMPs) are also considered important virulence factors that induce autophagy. The induction of autophagy by bacterial PAMPs has been discussed in the section on autophagy in innate immunity.

\section{Autophagic elimination of bacteria}

Autophagy has been demonstrated to be a key defense mechanism for the control of bacterial infection both in vitro and in vivo. Extensive work has been done to determine the mechanisms of xenophagic elimination of bacteria. ${ }^{55}$ Autophagy limits the growth of diverse species of bacteria, including Group A Streptococcus (GAS), Mycobacterium tuberculosis, Rickettsia conorii, Salmonella typhimurium, and $S$. flexneri. ${ }^{56,57}$ The pathway can target intracellular bacteria, within phagosomes (eg, M. tuberculosis), in damaged vacuoles (eg, $S$. enterica serovar Typhimurium), or in the cytosol (eg, GAS), and kill them via the autolysosome.

Though typically extracellular bacteria, GAS can enter the cytosol of host cells when internalized into endosomes, which are then captured by autophagosomes. GAS-containing autophagosomes have been found to eventually fuse with lysosomes, resulting in killing of most intracellular GAS and preventing GAS replication. ${ }^{58}$

Studies have demonstrated that stimulation of autophagy suppressed the intracellular survival of $M$. tuberculosis in vitro. ${ }^{59}$ Upon infection of macrophages, M. tuberculosis blocks phagosomal maturation in order to survive. Induction of autophagy facilitates mycobacterial phagosome fusion with lysosomes and degradation of the pathogen. ${ }^{59}$ Furthermore, M. tuberculosis infection of autophagy-gene-deficient conditional knockout mice resulted in increased bacterial burden as well as excessive tissue inflammation compared to 


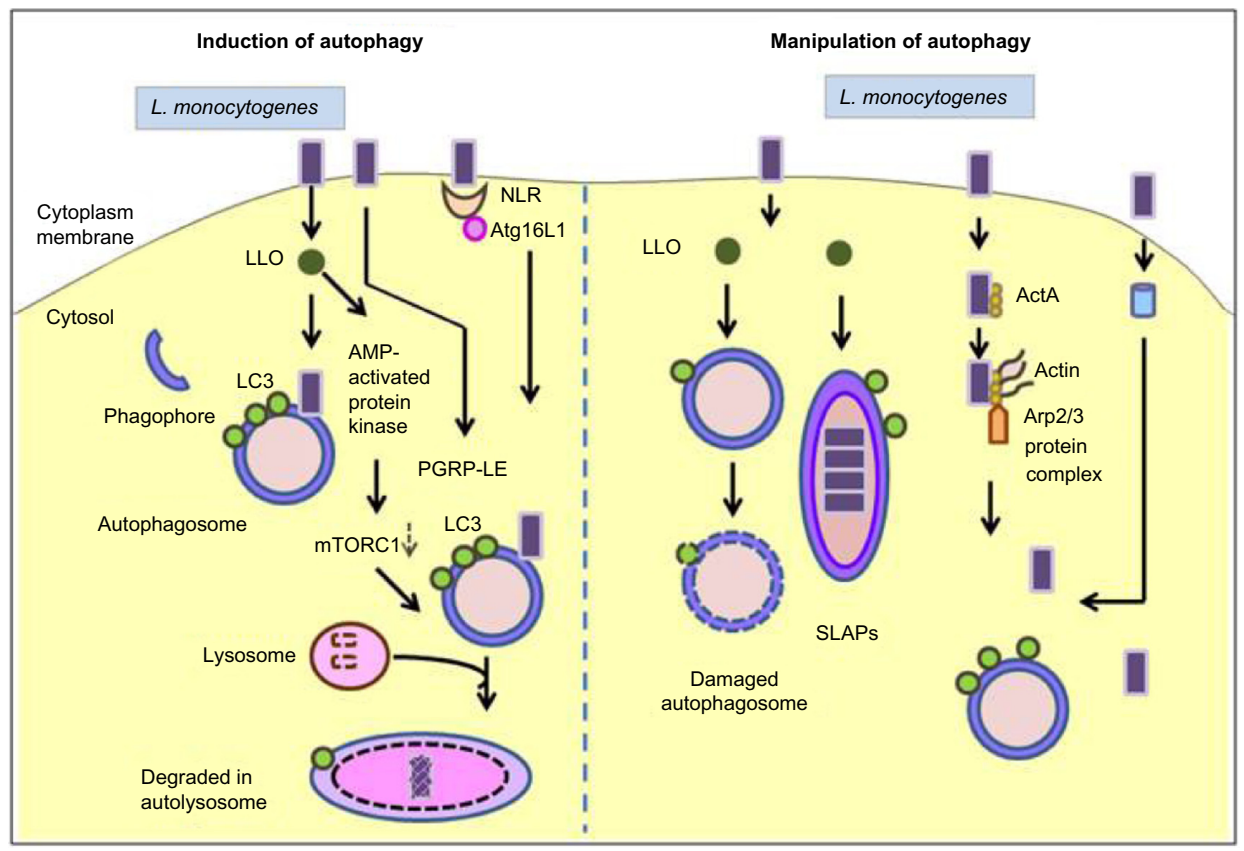

Figure 2 Interaction of autophagy with Listeria monocytogenes.

Notes: At the early stage of infection, Listeria induces autophagy via LLO, activation of a peptidoglycan-recognition protein member, PGRP-LE, NODI, and NOD2. At a later stage of infection, Listeria utilizes several virulence factors, including LLO, InIK, and the actin polymerization protein ActA to avoid entrapment in autophagosomes. Abbreviations: LLO, listeriolysin O; SLAPs, spacious Listeria-containing phagosomes; NOD, nucleotide-binding oligomerization domain.

autophagy-proficient littermates. ${ }^{60}$ Thus, autophagy in vivo is important not only in bacterial clearance but also in prevention of host tissue destruction.

On the other, phagolysosomal killing can also occur through the alternate mechanism of LC3-associated phagocytosis (LAP). As opposed to canonical autophagy, in this case, following the uptake of an invading bacterium by conventional phagocytosis, the autophagy machinery enhances the maturation of the phagosomes through Beclin1-VP34 complexes and LC3 conjugation systems, independently of ULK $1 .^{4}$

\section{Bacterial manipulation of autophagy}

In order to survive in host cells, intracellular bacteria have evolved mechanisms to evade (eg, Shigella flexneri), inhibit (eg, Legionella pneumophila), and subvert (Coxiella burnetii) autophagy. Cytosolic L. monocytogenes utilizes several virulence factors, including LLO and the actin polymerization protein ActA, to avoid entrapment in autophagosomes. ${ }^{52}$ LLO damages the membrane of autophagosomes. Expression of ActA on the bacterial surface recruits the host cell proteins Arp2/3 complex and actin, which help prevent marking of the bacteria by ubiquitination and recognition by components of the autophagic pathway. ${ }^{61,62}$ L. monocytogenes can replicate in LAMP1-positive spacious Listeria-containing phagosomes
(SLAPs), the formation of which is dependent on both LLO and the host autophagy pathway ${ }^{63}$ (Figure 2). Most recently, it was reported that L. monocytogenes hijacks the host major vault protein through interaction with InIK, a listerial virulence factor, thus preventing their ubiquitination and escape from autophagic recognition. ${ }^{64}$ Two bacterial phospholipase C (PLC) enzymes, with substrate preferences for phosphatidylinositol (PI-PLC) or phosphatidylcholine and other phosphoinositides (PC-PLC), may mediate autophagy evasion by disrupting the inner membrane of the autophagosomes. ${ }^{52}$ Mutant bacteria lacking PI-PLC or PC-PLC expression were targeted by autophagy at later times during infection.

In contrast to the bacteria that try to evade autophagic elimination, certain bacteria actively exploit autophagy to support creation of the specialized vacuole in which they replicate. C. burnetii survives in large Coxiella-replicative vacuoles (CRVs) that are decorated with the autophagy components LC3 and Beclin1. Overexpressing LC3 or Beclin1 promotes bacterial infection and increases the number and size of the CRVs during early infection, while the inhibition of autophagy impairs CRV formation and bacterial replication. ${ }^{65,66}$ Anaplasma phagocytophilum actively induces autophagy by secreting Anaplasma translocated substrate 1 (Ats-1), a type IV secretion effector, which hijacks the Beclin1-Atg14L autophagy initiation pathway. ${ }^{67}$ Stimulation of autophagy with rapamycin facilitates $A$. phagocytophilum 
infection, which may mean that the autophagosome provides Anaplasma with direct access to host cytosolic nutrients without the need for transport across the inclusion membrane. ${ }^{68}$

\section{Autophagy in innate immunity}

Autophagy has been widely recognized as an important innate immune mechanism due to its role in pattern recognition receptor (PRR) recognition of pathogen components and in regulation of type I IFN induction pathways. These functions are mediated through feedback loops by which autophagy either upregulates the activation of type I interferon (IFN) responses or downregulates type I IFN signaling following a period of productive induction. ${ }^{69}$ Pathogen recognition is the first step of innate immunity. The response to pathogens by the innate immune system is initiated through the detection of PAMPs by a variety of host PRRs. Among the cellular PRRs, the toll-like receptors (TLRs) are the first class of PRRs that were associated to autophagy. TLR engagement with their cognate ligand triggers the production of cytokines. TLRs are membrane-bound proteins that are expressed predominantly in intracellular endosomal compartments. Autophagy assists TLRs in meeting their cognate ligands by sequestering the cytosolic PAMPs and delivering them to the endosomally located and luminally oriented TLRs ${ }^{69,70}$ (Figure 3 ).

Autophagy has also emerged as an important player in regulating innate immune responses induced through the alternate PRRs, the RIG-I-like receptors (RLRs), which recognize dsRNA and the sensors of intracellular DNA. Mitochondria serve as coordinating sites of RLR signaling, and activation of autophagic processes regulates RLR signaling, by promoting clearance of reactive oxygen species (ROS)-containing dysfunctional mitochondria. Further, the Atg5-Atg12 conjugate, a key factor of autophagy, negatively regulates the type I IFN signaling by direct association with RLR and IFN signaling by direct association with RLR and mitochondrial antiviral signaling protein (MAVS). ${ }^{70}$ Additionally, autophagy has also been implicated in the turnover of the ER-associated adaptor, stimulator of interferon genes (STING), an important transducer of the innate signaling response. Atg9, a key protein in the autophagosome membrane, regulates the assembly of TBK1 with STING after dsDNA sensing ${ }^{69}$ (Figure 4).

Activation of both nucleotide-binding oligomerization domain 1 (NOD1) and NOD2 by NOD-like receptors (NLRs) activates autophagy by recruiting Atg16L1 to the plasma membrane at the entry site of the invading L. monocytogenes, leading to their efficient sequestration in autophagosomes and subsequent killing. ${ }^{71}$ Further, it has been demonstrated that a cytosolic PRR, a peptidoglycan-recognition protein (PGRP) member, PGRP-LE, which recognizes diaminopimelic acid-type peptidoglycan, induces autophagy. PGRP-LE is crucial for autophagy targeting of Listeria in Drosophila

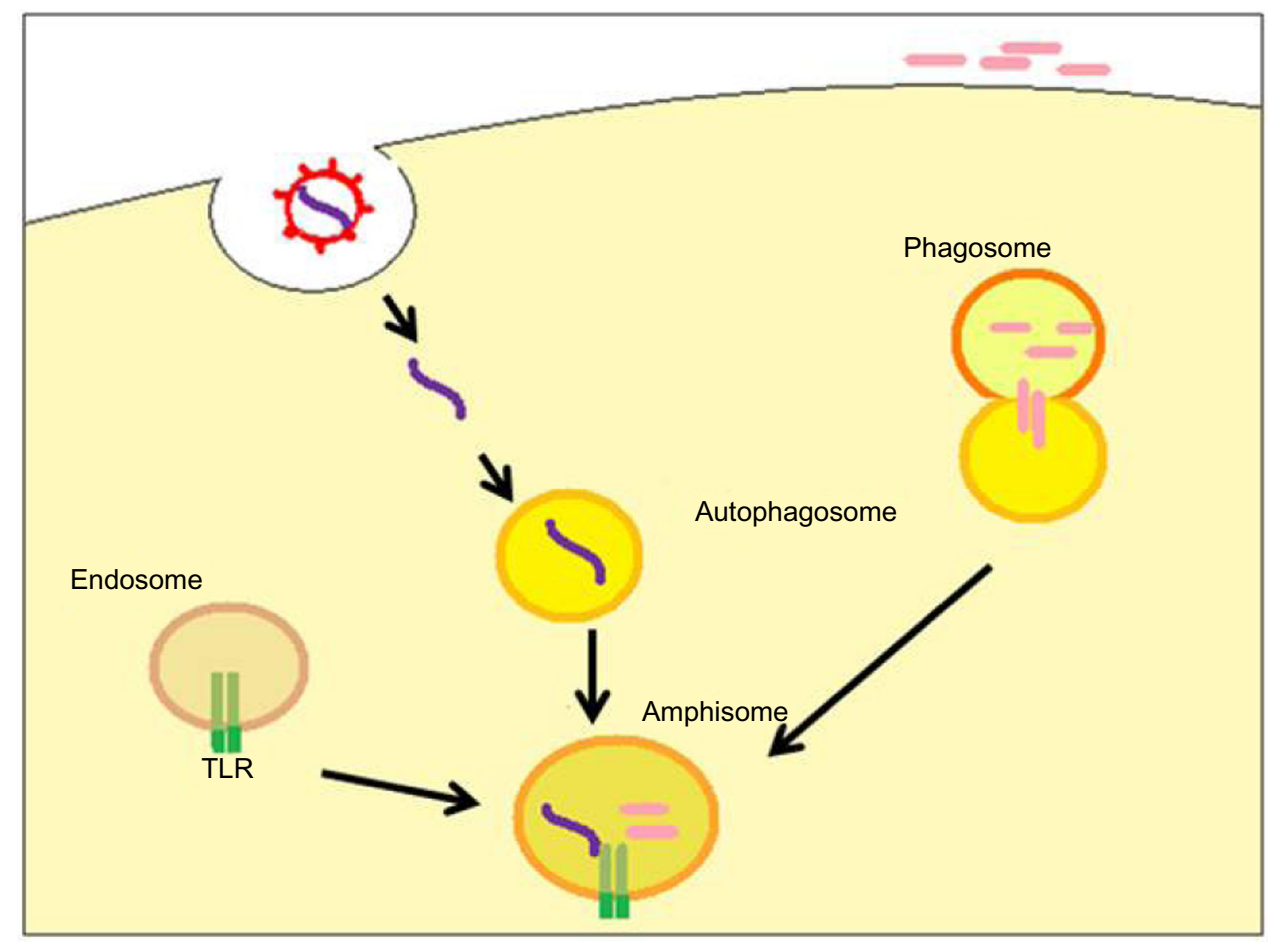

Figure 3 Autophagy promotes pathogen sensing by promoting delivery of pathogen-associated molecular patterns (PAMPs) to the endosomal toll-like receptors (TLR)s. 


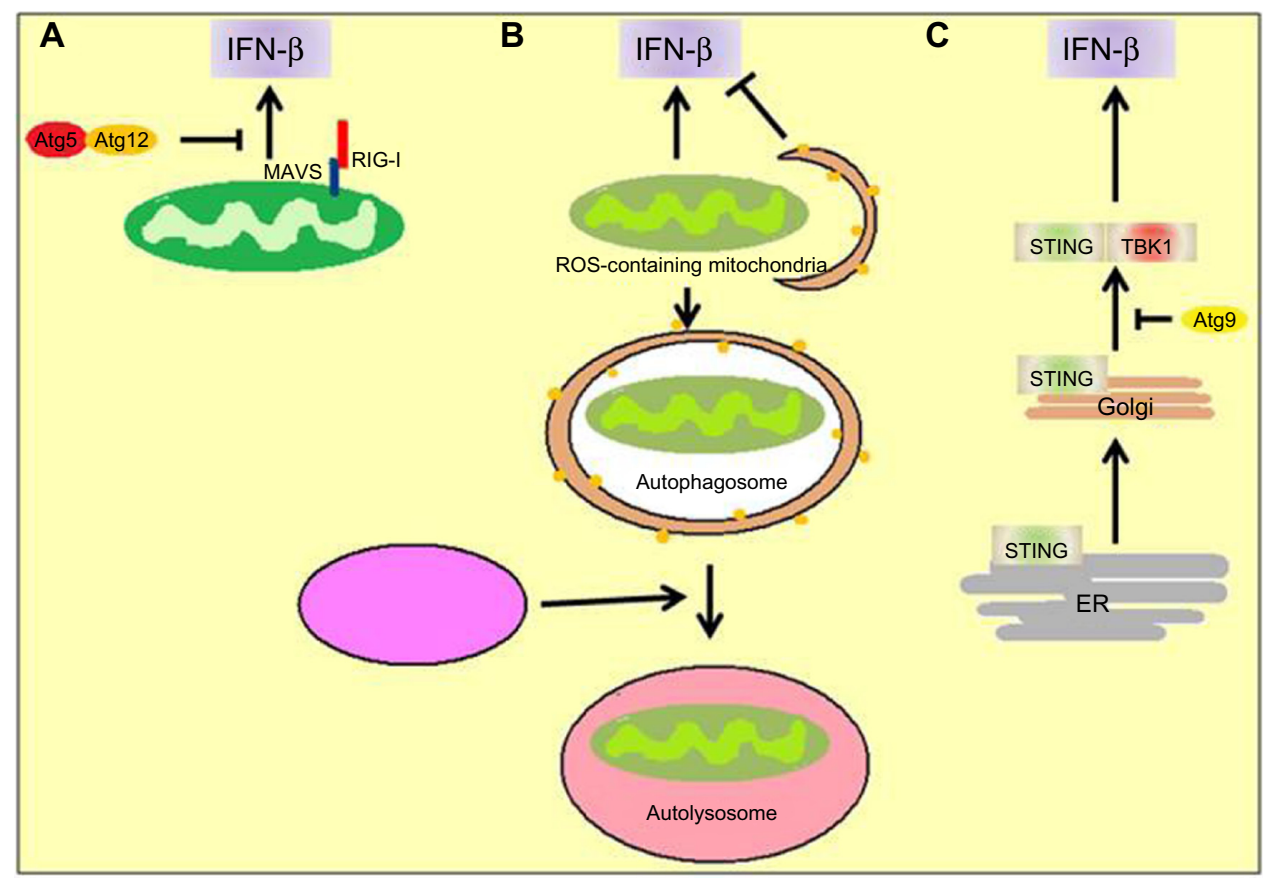

Figure 4 Autophagy negatively regulates type I interferon induction through multiple mechanisms.

Notes: (A) The Atg5-Atg12 complex blocks RLR-MAVS interaction. (B) Mitophagy eliminates reactive oxygen species (ROS)-containing mitochondria. (C) Atg9 controls the assembly of stimulator of interferon genes (STING) with TBKI following its translocation from the endoplasmic reticulum (ER) to Golgi.

melanogaster and therefore protects the fruit fly from Listeria monocytogenes infection. ${ }^{72}$

On the other hand, autophagy has also been suggested to be one of the downstream effectors by which TLR3 mediates elimination of invading viruses, and Beclin1 is a key initiator of this response. Infection with HIV, a virus that activates TLR7/8, induced autophagy in HeLa cells. Conversely, vesicular stomatitis virus (VSV) infection failed to upregulate autophagy in plasmacytoid dendritic cells (DCs), and the failure was attributed to high baseline autophagy in DCs. ${ }^{69}$ Previously, we showed that the type I IFN subtypes IFN- $\alpha$ and IFN- $\beta$ induce a differential autophagic sequestration of ER and/or mitochondria-associated proteins through the RLR signaling adaptor MAVS, and only a high relative induction of IFN- $\beta$ exclusively favors autolysomal degradation of the sequestered proteins. ${ }^{73}$ Alternatively, IFN- $\gamma$ induces macroautophagy and mycobacterial clearance through IRGs. It has also been reported to enhance $M$. tuberculosis and Ricksettia conorii degradation by autophagy in infected cells. ${ }^{74}$ The Atg5-Atg12/Atg16L1 complex performs a pivotal, nondegradative role in IFN- $\gamma$-mediated antiviral defense, establishing that multicellular organisms have evolved to selectively use portions of the autophagy machinery for host defense. IFN- $\gamma$, via Atg5-Atg12/Atg16L1, inhibited the formation of the membranous cytoplasmic murine norovirus (MNV) replication complex, where Atg16L1 localized. ${ }^{75}$
Recently, a paradigm has emerged in which Th1 cytokines induce autophagy, while Th2 cytokines inhibit autophagy. ${ }^{76}$

Tumour necrosis factor (TNF)- $\alpha$ upregulates autophagy in cells lacking NF- $\kappa \mathrm{B}$ activation. The TNF-related apoptosis-inducing ligand (TRAIL) has been described to induce autophagy in human epithelial cells, and the TRAIL induction of autophagy is regulated through the inactivation of Fas-associated death domain (FADD), the signaling adapter protein of the TRAIL receptor. Likewise, the CD40 ligand, also a TNF family member, has been shown to induce autophagy-mediated fusion of Toxoplasma gondii-containing phagosomes with lysosomes through CD40 signaling in macrophages. $^{74}$

\section{Autophagy and adaptive immunity}

Autophagy enables the immune surveillance for intracellular antigens by aiding the induction and execution of adaptive immune responses. MHC class II protein expression is induced during a type I IFN signaling response. MHC class II subunits assemble in the ER and transit to endosomal compartments. Studies have shown that autophagy is involved in the MHC class II processing and presentation of various intracellular Ags to CD4 ${ }^{+} \mathrm{T}$ cells (Figure 5). Physical intersection of autophagy pathways with endosomes and lysosomes is critical in promoting cytosolic and nuclear Ag processing and presentation by MHC class II molecules. The MHC class II 


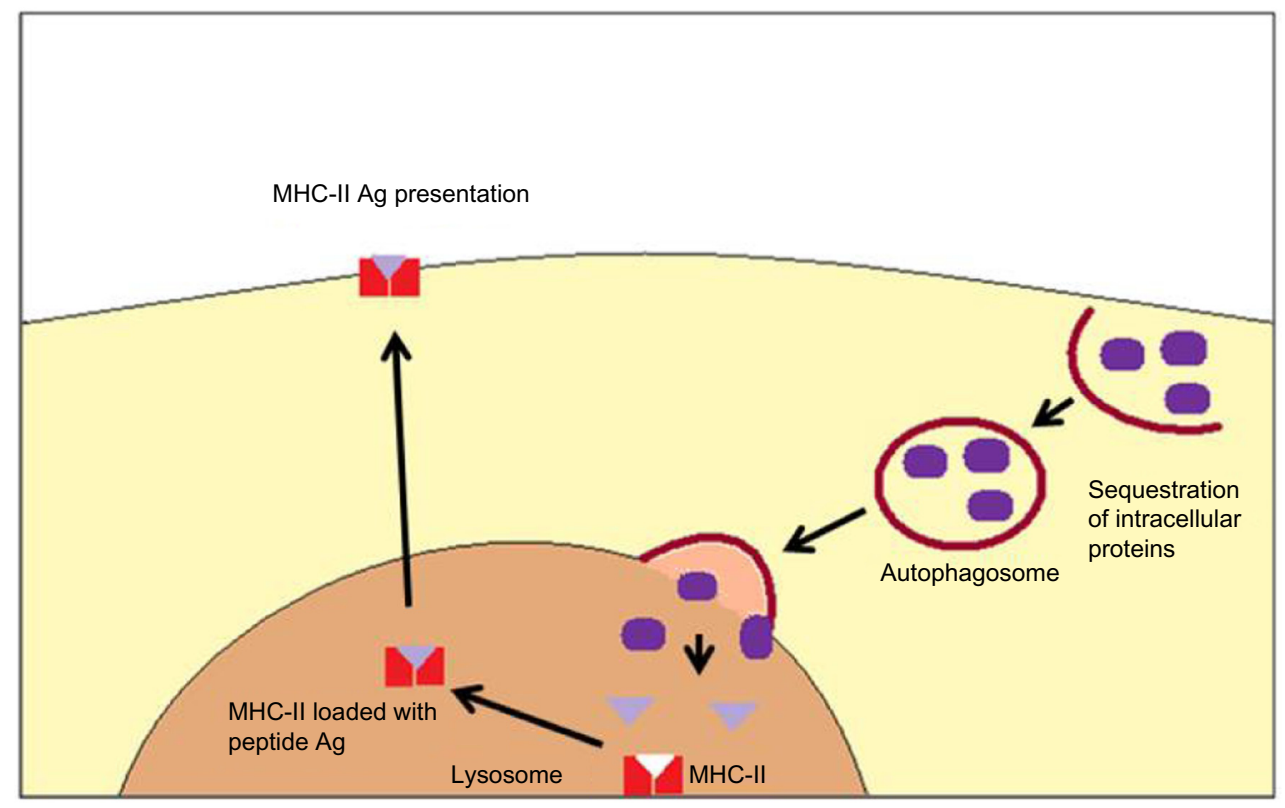

Figure 5 Autophagy governs the processing and presentation of intracellular antigens by MHC-II complexes.

Note: Intracellular and phagocytosed proteins undergo autolysosomal processing and are mounted onto MHC-Il complexes for presentation to CD4+ T cells.

complexes travel through early endosomes, multivesicular bodies, and lysosomes to acquire peptides before transport to the cell surface for display to $\mathrm{CD} 4^{+} \mathrm{T}$ cells.${ }^{77} \mathrm{MHC}$ class II Ag presentation was inhibited on treatment with PI3 kinase inhibitors that block the induction of autophagy and upregulated upon treatment with low doses of the lysosomotropic agent ammonium chloride. ${ }^{78}$ The EBV EBNA1 protein is known to be presented on MHC class II through autophagy. On the other hand, the HIV envelope proteins are known to subvert MHC class II presentation by enhancing mTOR signaling. ${ }^{78}$ Exposure to TLR ligands has been shown to regulate autophagy pathways as well as cellular endocytosis. Studies on HSV have suggested a link between innate signaling via TLR and $\mathrm{Ag}$ cross-presentation. In vivo activation of $\mathrm{CD}^{+} \mathrm{T}$ cells was significantly impaired in HSV-infected Atg5 knockout mice. Atg5-deficient DCs when infected with HSV failed to prime $\mathrm{CD} 4^{+} \mathrm{T}$ cells, clearly indicating a role of autophagy in Ag cross-presentation. ${ }^{77} \mathrm{EBV}$-infected pDC are unable to activate a full T-cell response, and this defect is attributed to the inhibition of TLR9 expression by the LMP1 oncoprotein of the virus. ${ }^{79}$ In the case of respiratory syncytial virus (RSV) infection, the synergism between TLR signaling and MHC class II Ag presentation in DCs was shown to be mediated through Beclin1. ${ }^{80}$ Similarly, NOD2-mediated autophagy in DCs is required for the generation of $\mathrm{CD} 4^{+} \mathrm{T}-$ cell responses during bacterial infections like $S$. enterica serovar typhimurium and Crohn's-associated, adherent-invasive E. coli. ${ }^{81}$ Further, autophagy enables host macrophages to compensate for bacterial inhibition of the endosomal MHC class II antigen presentation pathway to mount a $\mathrm{CD} 4^{+} \mathrm{T}-$ cell response against Yersinia pseudotuberculosis. ${ }^{82}$

Intracellular cytosolic or nuclear Ags are presented to $\mathrm{CD}^{+} \mathrm{T}$ cells by MHC class I molecules generally after proteosomal hydrolysis. In contrast, viruses are also known to induce alternate pathways of MHC class I Ag presentation and CD8 responses through autophagy (Figure 6). In the late stages of HSV infection, viral capsid presentation is dependent on $\mathrm{Ag}$ processing in the lysosomal compartments as well as on Atg5 and is impaired by the viral ICP34.5-mediated inhibition of autophagy. ${ }^{77}$ Similarly, the MHC class I presentation of an HCMV epitope derived from the viral pUL138 latency-associated protein was shown to be mediated by an autophagy-dependent mechanism, and chemical inhibition of autophagy or Atg12 silencing inhibited the stimulation of pUL138 Ag-specific CD8 ${ }^{+} \mathrm{T}$ cells. Moreover, the Ag itself was found to be localized with LC3, LAMP-2, and endocytosed MHC I. Additionally, DCs can also cross-present Ags from apoptotic cells to activate MHC I-restricted CTLs. It has been shown that autophagy in dying IAV PR8/34-infected cells potentiates the cross-presentation of IAV Ags by DCs, leading to the induction of a robust anti-IAV cytotoxic response, in vivo. Likewise, autophagic processing also plays a role in intracellular Chlamydia epitope MHC I presentation. Interestingly, tranporter associated with antigen processing (TAP) and MHC I are colocalized to autophagosomes following DC infection with Chlamydia. The autophagic 


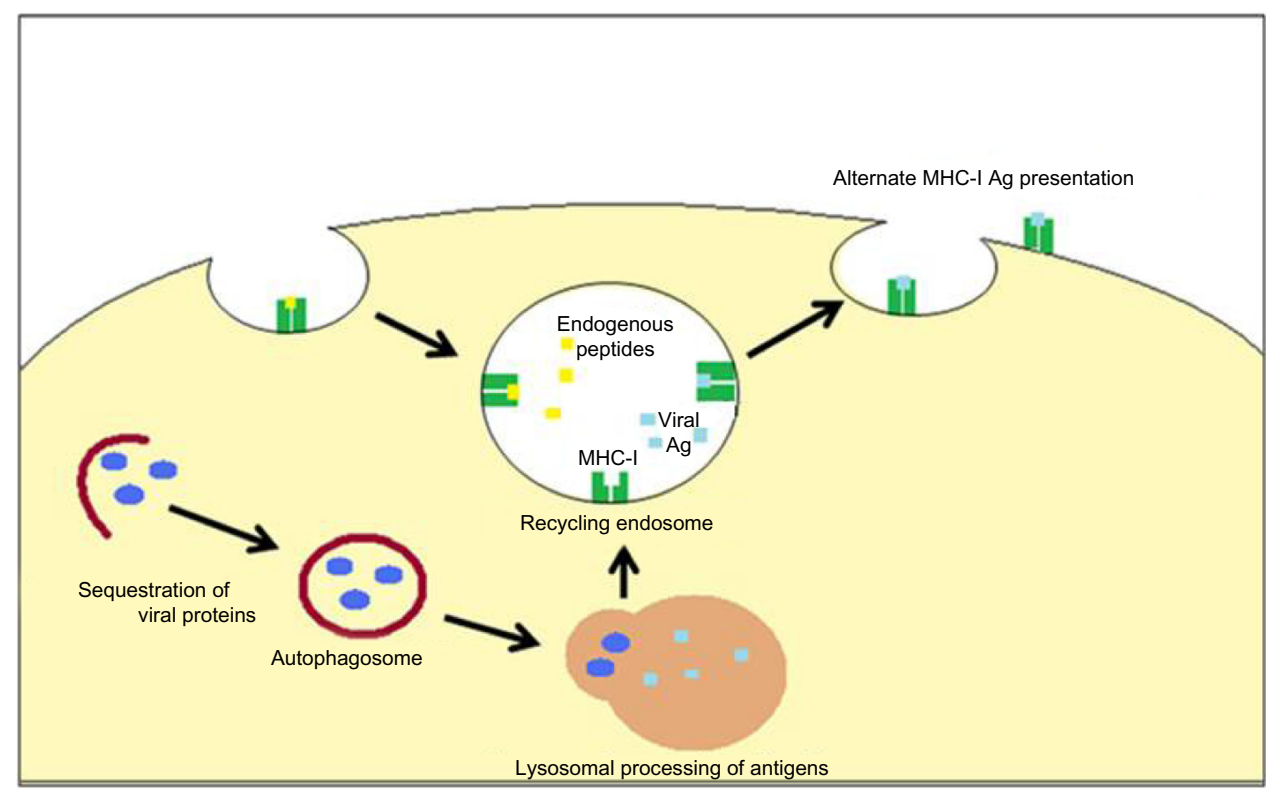

Figure 6: Autophagy facilitates processing and loading of sequestered cytosolic proteins to MHC-I complexes.

Notes: Recycling endosomes capture MHC-I and fuse with autolysosomes to serve as sites for peptide exchange, so as to allow the loading of lysosomally processed viral proteins to $\mathrm{MHC}-\mathrm{I}$ complexes and subsequent presentation to $\mathrm{CD} 8^{+} \mathrm{T}$ cells.

targeting allows preprocessing of the bacterial antigens in the lysosomes, which is then followed by their cytosolic release and further processing by the proteasome before loading onto recycling MHC I complexes. ${ }^{5}$

Additionally, autophagy is involved in multiple aspects of lymphocyte development and function and is essential for both $\mathrm{T}$ and $\mathrm{B}$ lymphocyte survival and proliferation. ${ }^{83}$ The pathway is highly induced in effector $\mathrm{T}$ cells and has been shown to promote the cytokine-dependent survival of primary $\mathrm{T}$ cells. In contrast, studies also suggest that autophagy is an important death pathway in T cells lacking FADD activity, caspase-8, or immunity-related GTPase family $M$ protein (IRGM)-1. Thus, autophagy has been shown to have both pro-survival and pro-death roles in T cells. ${ }^{84}$ On the other hand, Atg5 and an intact autophagy pathway are required at specific stages in B-cell development and differentially required for distinct, but closely related, cell lineages. ${ }^{85}$

\section{Autophagic regulation of inflammation}

Autophagy not only plays a role in pathogen sensing and restriction but also has many other functions in the immune system, including processing of PAMPs for PRR recognition, inflammasome regulation, and unconventional secretion of alarmins. ${ }^{70,86}$ Inflammasomes are protein complexes that respond to PAMPs and damage-associated molecular patterns (DAMPs) by inducing proteolytic processing and secretion of IL-1 $\beta$ and IL-18. Increasing evidence from various studies ${ }^{86-90}$ supports that autophagy negatively regulates inflammasome activation. It has been suggested that basal levels of autophagy control the set point of inflammasome activation by clearing cytosolic debris, protein aggregates, and defective organelles. ${ }^{4}$ More specifically, autophagy has been reported to control the production of IL-1 $\beta$ through at least two separate mechanisms: by targeting pro-IL-1 $\beta$ for lysosomal degradation, and by regulating activation of the NLRP3 inflammasome. Following treatment of macrophages with TLR ligands, pro-IL-1 $\beta$ was seen to be sequestered in autophagosomes, whereas specific activation of autophagy with rapamycin induced the degradation of pro-IL-1 $\beta$ and blocked secretion of the mature cytokine. Conversely, the inhibition of autophagy promoted the secretion of IL-1 $\beta$ by macrophages in a NLRP3- and TRIF-dependent fashion. ${ }^{87}$ Similarly, autophagy inhibition in dendritic cells also promotes the secretion of both IL-1 $\beta$ and IL-23, and supernatants from these cells stimulated the innate secretion of IL-17, IFN- $\gamma$, and IL-22 by $\gamma \delta$ T cells. ${ }^{88}$ In vivo, the deficiency of Atg16L1 represents a source of sterile inflammation that leads to inflammasome activation and increased IL-1 $\beta$ processing. Further, autophagy inhibits the cytosolic release of NALP3 inflammasome-mediated mitochondrial DNA, which is an endogenous source of inflammasome agonists. ${ }^{89}$ In contrast, a proinflammatory export pathway that mediates an unconventional secretion of IL-1 $\beta$, IL-18 and the DAMP HMGB1 depends on specific Atg factors and the mammalian Golgi reassembly stacking protein (GRASP) paralogue, GRASP55. ${ }^{4}$ 
Additionally, autophagy also mediates degradation of other proinflammatory factors such as the NF- $\kappa B$ signaling components, NIKs (NF- $\mathrm{KB}$-inducing kinases), and the IKK (the inhibitor of NF- $\mathrm{B}$ ) protein family, and complex interactions have been reported between both pathways. ${ }^{90}$ With respect to viral infection, the murine cytomegalovirus (MCMV) M45 protein binds to the NF- $\kappa \mathrm{B}$ subunit NEMO targeting it for autophagic degradation. In contrast, TNFdependent activation of NF- $\kappa \mathrm{B}$ represses autophagy through the activation of mTOR. In macrophages exposed to E. coli, the suppression of prolonged $\mathrm{NF}-\kappa \mathrm{B}$ activity promotes

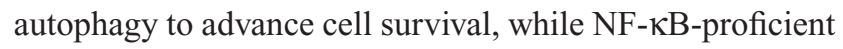
macrophages undergo cell death under the same conditions. Defects in the autophagic response can lead to inflammatory and autoimmune disorders. ${ }^{4,5,90}$

\section{Concluding remarks}

As the role of autophagy in eukaryotic cells has evolved much beyond its basic metabolic function, the pathway appears to be integrated with all stages of antimicrobial host defense. Not surprisingly, thus, pathogens have devised strategies to evade as well as exploit the process for their survival and proliferation. It is becoming increasingly evident that the pathway plays a vital role in determining the disease course of infection. In light of the intricate interplay between autophagy and pathogens, the pathway has often been suggested as a target for new interventional approaches against infectious diseases. However, its dichotomous role in limiting as well as in favoring the propagation of pathogens and its involvement in a range of biologic processes could complicate its therapeutic application. The difficulties are further compounded by the fact that stimuli that activate autophagy also trigger other stress responses. Thus, extreme dissection of the molecular mechanisms underlying the pathogen-autophagy interactions is warranted for the selective harnessing of the host-beneficial potential of the response.

\section{Acknowledgments}

We apologize to all researchers in the field whose work has not been cited and in some cases having cited reviews instead of primary articles due to space limitations. The work was supported by NIHAI109100.

\section{Disclosure}

The authors report no conflicts of interest in this work.

\section{References}

1. Rubinsztein DC, Codogno P, Levine B. Autophagy modulation as a potential therapeutic target for diverse diseases. Nat Rev Drug Discov. 2012;11:709-730.
2. Dreux M, Chisari FV. Viruses and the autophagy machinery. Cell Cycle. 2010;9(7):1295-1307.

3. Kraft C, Kijanska M, Kalie E, et al. Binding of the Atg1/ULK1 kinase to the ubiquitin-like protein Atg8 regulates autophagy. EMBO J. 2012;31:3691-3703.

4. Deretic V, Saitoh T, Akira S. Autophagy in infection, inflammation and immunity. Nat Rev Immunol. 2013;13:722-737.

5. Perot BP, Ingersoll MA, Albert ML. The impact of macroautophagy on CD8(+) T-cell-mediated antiviral immunity. Immunol Rev. 2013;255: 40-56.

6. Tang D, Kang R, Coyne CB, Zeh HJ, Lotze MT. PAMPs and DAMPs: signal 0 s that spur autophagy and immunity. Immunol Rev. 2012;249: 158-175.

7. Cavignac Y, Esclatine A. Herpesviruses and autophagy: catch me if you can! Viruses. 2010;2:314-333

8. Kampa-Schittenhelm KM, Heinrich MC, Akmut F, et al. Cell cycledependent activity of the novel dual PI3K-MTORC1/2 inhibitor NVPBGT226 in acute leukemia. Mol Cancer. 2013;12:46.

9. Efeyan A, Zoncu R, Chang S, et al. Regulation of mTORC1 by the Rag GTPases is necessary for neonatal autophagy and survival. Nature. 2013;493:679-683.

10. Alers S, Loffler AS, Wesselborg S, Stork B. Role of AMPK-mTORUlk $1 / 2$ in the regulation of autophagy: cross talk, shortcuts, and feedbacks. Mol Cell Biol. 2012;32:2-11.

11. Hasty P, Sharp ZD, Curiel TJ, Campisi J. mTORC1 and p53: clash of the gods? Cell Cycle. 2012;12:20-25.

12. Cabrera R, Tu Z, Xu Y, et al. An immunomodulatory role for CD4(+) CD25(+) regulatory $\mathrm{T}$ lymphocytes in hepatitis $\mathrm{C}$ virus infection. Hepatology. 2004;40:1062-1071.

13. Jheng JR, Ho JY, Horng JT. ER stress, autophagy, and RNA viruses. Front Microbiol. 2014;5:388.

14. Pengo N, Scolari M, Oliva L, et al. Plasma cells require autophagy for sustainable immunoglobulin production. Nat Immunol. 2013;14:298-305.

15. Deretic V. Autophagy in immunity and cell-autonomous defense against intracellular microbes. Immunol Rev. 2011;240:92-104.

16. Levine B, Mizushima N, Virgin HW. Autophagy in immunity and inflammation. Nature. 2011;469:323-335.

17. Deretic V. Autophagy in innate and adaptive immunity. Trends Immunol. 2005;26:523-528.

18. Kirkegaard K, Jackson WT. Topology of double-membraned vesicles and the opportunity for non-lytic release of cytoplasm. Autophagy. $2005 ; 1: 182-184$.

19. Dong X, Levine B. Autophagy and viruses: adversaries or allies? J Innate Immun. 2013;5:480-493.

20. Jordan TX, Randall G. Manipulation or capitulation: virus interactions with autophagy. Microbes Infect. 2012;14:126-139.

21. Dreux M, Chisari FV. Impact of the autophagy machinery on hepatitis C virus infection. Viruses. 2011;3:1342-1357.

22. Ke PY, Chen SS. Autophagy in hepatitis C virus-host interactions: potential roles and therapeutic targets for liver-associated diseases. World J Gastroenterol. 2014;20:5773-5793.

23. Kim SJ, Syed GH, Khan M, et al. Hepatitis C virus triggers mitochondrial fission and attenuates apoptosis to promote viral persistence. Proc Natl Acad Sci U S A. 2014;111:6413-6418.

24. Kirkegaard K. Subversion of the cellular autophagy pathway by viruses. Curr Top Microbiol Immunol. 2009;335:323-333.

25. Taylor MP, Kirkegaard K. Potential subversion of autophagosomal pathway by picornaviruses. Autophagy. 2008;4:286-289.

26. Shi J, Luo H. Interplay between the cellular autophagy machinery and positive-stranded RNA viruses. Acta Biochim Biophys Sin (Shanghai). 2012;44:375-384

27. Richards AL, Jackson WT. Intracellular vesicle acidification promotes maturation of infectious poliovirus particles. PLoS Pathog. 2012;8:e1003046.

28. Richards AL, Jackson WT. That which does not degrade you makes you stronger: infectivity of poliovirus depends on vesicle acidification. Autophagy. 2013;9:806-807. 
29. Kemball CC, Alirezaei M, Flynn CT, et al. Coxsackievirus infection induces autophagy-like vesicles and megaphagosomes in pancreatic acinar cells in vivo. J Virol. 2010;84:12110-12124.

30. Robinson SM, Tsueng G, Sin J, et al. Coxsackievirus B exits the host cell in shed microvesicles displaying autophagosomal markers. PLoS Pathog. 2014;10:e1004045.

31. Wurdinger T, Gatson NN, Balaj L, Kaur B, Breakefield XO, Pegtel DM. Extracellular vesicles and their convergence with viral pathways. Adv Virol. 2012;2012:767694.

32. Beale R, Wise H, Stuart A, Ravenhill BJ, Digard P, Randow FA. LC3-interacting motif in the influenza A virus M2 protein is required to subvert autophagy and maintain virion stability. Cell Host Microbe. 2014;15:239-247.

33. Zhirnov OP, Klenk HD. Influenza A virus proteins NS 1 and hemagglutinin along with M2 are involved in stimulation of autophagy in infected cells. J Virol. 2013;87:13107-13114.

34. Gannagé M, Dormann D, Albrecht R, et al. Matrix protein 2 of influenza A virus blocks autophagosome fusion with lysosomes. Cell Host Microbe. 2009;6:367-380

35. Munz C. Beclin-1 targeting for viral immune escape. Viruses. 2011;3: 1166-1178.

36. Li JK, Liang JJ, Liao CL, Lin YL. Autophagy is involved in the early step of Japanese encephalitis virus infection. Microbes Infect. 2012;14 159-168.

37. Sharma M, Bhattacharyya S, Nain M, et al. Japanese encephalitis virus replication is negatively regulated by autophagy and occurs on LC3-I- and EDEM1-containing membranes. Autophagy. 2014;10(9): 1637-1651.

38. Tang SW, Ducroux A, Jeang KT, Neuveut C. Impact of cellular autophagy on viruses: Insights from hepatitis B virus and human retroviruses. J Biomed Sci. 2012;19:92.

39. Campbell GR, Spector SA. Inhibition of human immunodeficiency virus type-1 through autophagy. Curr Opin Microbiol. 2013;16:349-354.

40. Zhang HT, Chen GG, Hu BG, et al. Hepatitis B virus x protein induces autophagy via activating death-associated protein kinase. JViral Hepat. 2014;21:642-649.

41. Liu B, Fang M, Hu Y, et al. Hepatitis B virus X protein inhibits autophagic degradation by impairing lysosomal maturation. Autophagy. 2014;10:416-430.

42. Lazar C, Macovei A, Petrescu S, Branza-Nichita N. Activation of ERAD pathway by human hepatitis B virus modulates viral and subviral particle production. PLoS One. 2012;7:e34169.

43. Li J, Liu Y, Wang Z, et al. Subversion of cellular autophagy machinery by hepatitis B virus for viral envelopment. J Virol. 2011;85:6319-6333.

44. Lee DY, Lee J, Sugden B. The unfolded protein response and autophagy: herpesviruses rule! J Virol. 2009;83:1168-1172.

45. Hung $\mathrm{CH}$, Chen LW, Wang WH, et al. Regulation of autophagic activation by Rta of Epstein-Barr virus via ERK-kinase pathway. J Virol. 2014;88(20):12133-12145.

46. Pratt ZL, Zhang J, Sugden B. The latent membrane protein 1 (LMP1) oncogene of Epstein-Barr virus can simultaneously induce and inhibit apoptosis in B cells. J Virol. 2012;86:4380-4393.

47. Granato M, Santarelli R, Farina A, et al. Epstein-barr virus blocks the autophagic flux and appropriates the autophagic machinery to enhance viral replication. J Virol. 2014;88:12715-12726.

48. Silva LM, Jung JU. Modulation of the autophagy pathway by human tumor viruses. Semin Cancer Biol. 2013;23:323-328.

49. Cheng CY, Chi PI, Liu HJ. Commentary on the regulation of viral proteins in autophagy process. Biomed Res Int. 2014;2014:962915.

50. Chaumorcel M, Lussignol M, Mouna L, et al. The human cytomegalovirus protein TRS1 inhibits autophagy via its interaction with Beclin 1. J Virol. 2012;86:2571-2584.

51. Rikihisa Y. Glycogen autophagosomes in polymorphonuclear leukocytes induced by rickettsiae. Anat Rec. 1984;208:319-327.

52. Birmingham CL, Canadien V, Gouin E, et al. Listeria monocytogenes evades killing by autophagy during colonization of host cells. Autophagy. 2007;3:442-451.
53. Portnoy DA, Jacks PS, Hinrichs DJ. Role of hemolysin for the intracellular growth of Listeria monocytogenes. J Exp Med. 1988;167:1459-1471.

54. Kloft N, Neukirch C, Bobkiewicz W, et al. Pro-autophagic signal induction by bacterial pore-forming toxins. Med Microbiol Immunol. 2010;199:299-309.

55. Levine B. Eating oneself and uninvited guests: autophagy-related pathways in cellular defense. Cell. 2005;120:159-162.

56. Deretic V, Levine B. Autophagy, immunity, and microbial adaptations. Cell Host Microbe. 2009;5:527-549.

57. Walker DH, Popov VL, Crocquet-Valdes PA, Welsh CJ, Feng HM. Cytokine-induced, nitric oxide-dependent, intracellular antirickettsial activity of mouse endothelial cells. Lab Invest. 1997;76:129-138.

58. Nakagawa I, Amano A, Mizushima N, et al. Autophagy defends cells against invading group A Streptococcus. Science. 2004;306: 1037-1040.

59. Gutierrez MG, Master SS, Singh SB, Taylor GA, Colombo MI, Deretic V. Autophagy is a defense mechanism inhibiting BCG and Mycobacterium tuberculosis survival in infected macrophages. Cell. 2004;119:753-766.

60. Castillo EF, Dekonenko A, Arko-Mensah J, et al. Autophagy protects against active tuberculosis by suppressing bacterial burden and inflammation. Proc Natl Acad Sci U S A. 2012;109:E3168-E3176.

61. Ogawa M, Yoshikawa Y, Mimuro H, Hain T, Chakraborty T, Sasakawa C. Autophagy targeting of Listeria monocytogenes and the bacterial countermeasure. Autophagy. 2011;7:310-314.

62. Yoshikawa Y, Ogawa M, Hain T, et al. Listeria monocytogenes ActAmediated escape from autophagic recognition. Nat Cell Biol. 2009;11: 1233-1240.

63. Birmingham CL, Canadien V, Kaniuk NA, Steinberg BE, Higgins DE, Brumell JH. Listeriolysin O allows Listeria monocytogenes replication in macrophage vacuoles. Nature. 2008;451:350-354.

64. Dortet L, Mostowy S, Samba-Louaka A, et al. Recruitment of the major vault protein by InlK: a Listeria monocytogenes strategy to avoid autophagy. PLoS Pathog. 2011;7:e1002168

65. Gutierrez MG, Vázquez CL, Munafó DB, et al. Autophagy induction favours the generation and maturation of the Coxiella-replicative vacuoles. Cell Microbiol. 2005;7:981-993.

66. Vazquez CL, Colombo MI. Coxiella burnetii modulates Beclin 1 and Bcl-2, preventing host cell apoptosis to generate a persistent bacterial infection. Cell Death Differ. 2009;17:421-438.

67. Niu H, Xiong Q, Yamamoto A, Hayashi-Nishino M, Rikihisa Y. Autophagosomes induced by a bacterial Beclin 1 binding protein facilitate obligatory intracellular infection. Proc Natl Acad Sci USA. 2012;109:20800-20807.

68. Niu H, Yamaguchi M, Rikihisa Y. Subversion of cellular autophagy by Anaplasma phagocytophilum. Cell Microbiol. 2008;10:593-605.

69. Delgado M, Singh S, De Haro S, et al. Autophagy and pattern recognition receptors in innate immunity. Immunol Rev. 2009;227:189-202.

70. Deretic V. Autophagy as an innate immunity paradigm: expanding the scope and repertoire of pattern recognition receptors. Curr Opin Immunol. 2012;24:21-31.

71. Travassos LH, Carneiro LA, Ramjeet M, et al. Nod1 and Nod2 direct autophagy by recruiting ATG16L1 to the plasma membrane at the site of bacterial entry. Nat Immunol. 2010;11:55-62.

72. Yano T, Mita S, Ohmori H, et al. Autophagic control of listeria through intracellular innate immune recognition in drosophila. Nat Immunol. 2008;9:908-916.

73. Desai MM, Gong B, Chan T, et al. Differential, type I interferonmediated autophagic trafficking of Hepatitis $\mathrm{C}$ virus proteins in mouse liver. Gastroenterology. 2011;141:674-685, 685. e671-e676.

74. Lunemann JD, Munz C. Autophagy in CD4+ T-cell immunity and tolerance. Cell Death Differ. 2009;16:79-86.

75. Hwang S, Maloney NS, Bruinsma MW, et al. Nondegradative role of Atg5-Atg12/Atg16L1 autophagy protein complex in antiviral activity of interferon gamma. Cell Host Microbe. 2012;11:397-409.

76. Yordy BIA. Autophagy in Antiviral Immunity. 1st ed. Austin, TX: Landes Bioscience; 2013. 
77. Crotzer VL, Blum JS. Autophagy and adaptive immunity. Immunology. 2010;131:9-17.

78. Oh JE, Lee HK. Autophagy in innate recognition of pathogens and adaptive immunity. Yonsei Med J. 2012;53:241-247.

79. Zhang L, Sung JJ, Yu J, et al. Xenophagy in Helicobacter pyloriand Epstein-Barr virus-induced gastric cancer. J Pathol. 2014;233: $103-112$.

80. Reed M, Morris SH, Jang S, Mukherjee S, Yue Z, Lukacs NW. Autophagy-inducing protein beclin-1 in dendritic cells regulates CD4 $\mathrm{T}$ cell responses and disease severity during respiratory syncytial virus infection. J Immunol. 2013;191:2526-2537.

81. Cooney R, Baker J, Brain O, et al. NOD2 stimulation induces autophagy in dendritic cells influencing bacterial handling and antigen presentation. Nat Med. 2010;16:90-97.

82. Rüssmann H, Panthel K, Köhn B, et al. Alternative endogenous protein processing via an autophagy-dependent pathway compensates for Yersinia-mediated inhibition of endosomal major histocompatibility complex class II antigen presentation. Infect Immun. 2010;78: $5138-5150$.

83. Pua HH, He YW. Autophagy and lymphocyte homeostasis. Curr Top Microbiol Immunol. 2009;335:85-105.
84. Kovacs JR, Li C, Yang Q, et al. Autophagy promotes T-cell survival through degradation of proteins of the cell death machinery. Cell Death Differ. 2011;19:144-152.

85. Miller BC, Zhao Z, Stephenson LM, et al. The autophagy gene ATG5 plays an essential role in B lymphocyte development. Autophagy. 2008;4:309-314.

86. Kuballa P, Nolte WM, Castoreno AB, Xavier RJ. Autophagy and the immune system. Annu Rev Immunol. 2012;30:611-646.

87. Harris J, Hartman M, Roche C, et al. Autophagy controls IL-1beta secretion by targeting pro-IL-1beta for degradation. $J$ Biol Chem. 2011;286:9587-9597.

88. Peral de Castro C, Jones SA, Ní Cheallaigh C, et al. Autophagy regulates IL-23 secretion and innate T cell responses through effects on IL-1 secretion. J Immunol. 2012;189:4144-4153.

89. Nakahira K, Haspel JA, Rathinam VA, et al. Autophagy proteins regulate innate immune responses by inhibiting the release of mitochondrial DNA mediated by the NALP3 inflammasome. Nat Immunol. 2011;12:222-230.

90. Trocoli A, Djavaheri-Mergny M. The complex interplay between autophagy and NF-kappaB signaling pathways in cancer cells. Am J Cancer Res. 2011;1:629-649.

\section{Publish your work in this journal}

ImmunoTargets and Therapy is an international, peer-reviewed open access journal focusing on the immunological basis of diseases, potential targets for immune based therapy and treatment protocols employed to improve patient management. Basic immunology and physiology of the immune system in health, and disease will be also covered. In addition, the journal will focus on the impact of manage-

\section{Dovepress}

ment programs and new therapeutic agents and protocols on patient perspectives such as quality of life, adherence and satisfaction. The manuscript management system is completely online and includes a very quick and fair peer-review system, which is all easy to use. Visit http://www.dovepress.com/testimonials.php to read real quotes from published authors. 